\title{
A phenylthiazole derivative demonstrates efficacy on treatment of the cryptococcosis \& candidiasis in animal models
}

Nívea P Sá1, Caroline M Lima1, Julliana R A dos Santos², Marliete C Costa1, Patrícia P de Barros $^{3}$, Juliana C Junqueira ${ }^{3}$, Jéssica A Vaz ${ }^{4}$, Renata B Oliveira ${ }^{4}$, Beth B Fuchs ${ }^{5}$, Eleftherios Mylonakis ${ }^{5}$, Carlos A Rosa1, Daniel A Santos ${ }^{1}$ \& Susana Johann*,1

${ }^{1}$ Department of Microbiology, Institute of Biological Sciences, Universidade Federal de Minas Gerais, Belo Horizonte, MG, Brazil ${ }^{2}$ Postgraduate Program, Universidade CEUMA (UNICEUMA), São Luís, MA, Brazil

${ }^{3}$ Department of Biosciences \& Oral Diagnosis, Institute of Science \& Technology, Univ Estadual de São Paulo, São José dos Campos, São Paulo, Brazil

${ }^{4}$ Department of Pharmaceutical Products, Faculdade de Farmácia, Universidade Federal de Minas Gerais, Belo Horizonte, MG, Brazil ${ }^{5}$ Division of Infectious Diseases, Rhode Island Hospital, Alpert Medical School \& Brown University, Providence, RI, 02903, USA

*Author for correspondence: Tel.: +55 313349 7700; Fax: +55 313295 3115; sjohann@icb.ufmg.br

Aim: In this work we test 2-(2-(cyclohexylmethylene)hydrazinyl)-4-phenylthiazole (CHT) against Cryptococcus spp. and Candida albicans. Methods: The ability of CHT to act in biofilm and also to interfere with C. albicans adhesion was evaluated, as well as the efficiency of the CHT in cryptococcosis and candidiasis invertebrate and murine models. Results \& conclusion: In the present work we verified that CHT is found to inhibit Cryptococcus and C. albicans affecting biofilm in both and inhibited adhesion of Candida to human buccal cells. When we evaluated in vivo, CHT prolonged survival of Galleria mellonella after infections with Cryptococcus gattii, Cryptococcus neoformans or C. albicans and promoted a reduction in the fungal burden to the organs in the murine models. These results demonstrate CHT therapeutic potential.

Lay abstract: Candida spp. and Cryptococcus spp. cause thousands of deaths each year. In general, antifungal drugs have several limitations to their use, and there are a limited number of these drugs available to be used in the treatments of fungal diseases. This work contributes to the search for new antifungal drugs for the treatment of candidiasis and cryptococcosis, aiming in the future, after all necessary tests, to serve as a basis for the production of drugs that could be used in the treatment of patients with these fungal diseases.

\section{Graphical abstract:}

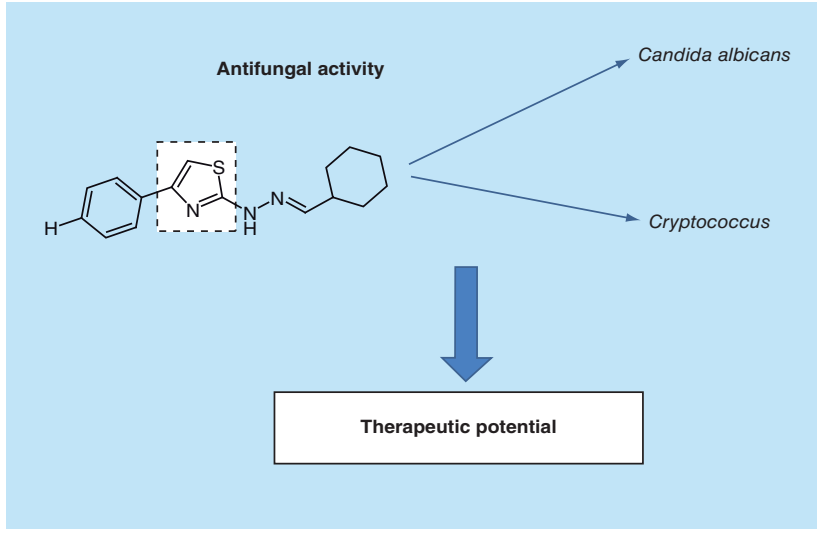

First draft submitted: 5 January 2018; Accepted for publication: 8 March 2018; Published online:

25 April 2018

Keywords: antifungal • Candida albicans • Cryptococcus spp. • Galleria mellonella • mice • thiazole • virulence 


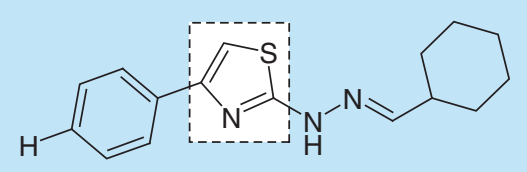

Figure 1. Chemical structure of 2-(2-(cyclohexylmethylene)hydrazinyl)-4-phenylthiazole, highlighting the thiazole portion.

Human body temperature provides a protective thermal barrier against the majority of environmental fungal species; however, about 300 species may cause disease in humans [1]. The most common pathogens that cause almost 90\% of deaths due to fungal disease are Candida spp., Aspergillus spp., and Cryptococcus spp. [2,3]. We will focus our discussion to the two pathogens: Candida albicans and Cryptococcus.

Cryptococcosis is responsible for more than one million cases and about 650,000 deaths per year worldwide, as an infectious disease with a universal geographical distribution, but clinical cases are more frequent in the Americas and Africa [3,4]. Cryptococcal infections occur by inhalation of the infectious propagules [5]. The opportunistic pathogen Cryptococcus neoformans is associated with immunodepression, while infection of immunocompetent hosts occurs primarily with Cryptococcus gattii [5,6]. Both forms of cryptococcosis, opportunistic and primary, are capable of causing severe and fatal meningoencephalitis, while the infection may involve organs such as the lung, skin, bones or kidneys [7].

C. albicans, the primary agent of candidiasis, is a commensal in healthy humans and can cause infections that are associated with staggeringly high mortality rates [8]. An estimated $24-70 \%$ of healthy people above 1 year of age are colonized by Candida and everyone is temporarily colonized at least once during their lifetime [9].

Amphotericin B, fluconazole and 5-fluorocytosine are antifungal agents most often used to treat patients with disseminated candidiasis and cryptococcosis [10]. Amphotericin B plus flucytosine combination is the treatment of choice during the induction phase in the treatment of cryptococcosis [11]. Unfortunately, amphotericin B is nephrotoxic, while flucytosine is not available in many countries and it is associated with bone marrow suppression and liver toxicity [11,12]. Maintenance therapy is supported by fluconazole, one of the major antifungal agents used in the treatment of cryptococcosis and disseminated candidiasis, as it has high efficacy and low toxicity [13]. However, one of the problems with fluconazole is the increasing incidence of fungal resistance [14,15].

Thus, a need remains to increase the drug arsenal of antifungal compounds. Our group has created some thiazoles derivatives with antifungal efficacy [16]. In the present work we evaluated the therapeutic potential of 2-(2-(cyclohexylmethylene)hydrazinyl)-4-phenylthiazole (CHT) against some of the prominent agents that lead to cryptococcosis and candidiasis.

\section{Materials \& methods Compound}

Thiazole compound CHT was synthesized according to methodology previously described by Sá et al. [16], previously designated as $1 \mathrm{~d}$ by these authors (Figure 1).

\section{Fungal strains}

C. neoformans ATCC24067 (AFLP2/VNIV) (= Cryptococcus deneoformans, according name proposed by Hagen et al. [17]) was obtained from the Culture Collection of the University of Georgia (GA, USA) and C. gattii L27/01 (VNII by PCR-fingerprinting using [GTG]5 primer, UnPUBlished DATA; = Cryptococcus deuterogattii, according name proposed by Hagen et al. [17]) from the Culture Collection of Microorganisms and Cells of the Universidade Federal of Minas Gerais under code UFMG-CM-Y6141 (Belo Horizonte, MG, Brazil). C. albicans SC5314, ATCC18804 and 10 C. albicans clinical isolates (UFMG-CM-Y6071, UFMG-CM-Y6072, UFMG-CMY6073, UFMG-CM-Y6074, UFMG-CM-Y6075, UFMG-CM-Y6076, UFMG-CM-Y6077, UFMG-CM-Y6080, UFMG-CM-Y6081 and UFMG-CM-Y6082), also deposited in the Culture Collection of Microorganisms and Cells of the Universidade Federal of Minas Gerais, were used in this study. Yeasts were grown on Sabouraud dextrose medium (Himedia, Mumbai, India) for 24 and $48 \mathrm{~h}$ for C. albicans and Cryptococcus spp., respectively, at $37^{\circ} \mathrm{C}$ prior to the experiments. All yeast strains were stored in glycerol at $-80{ }^{\circ} \mathrm{C}$. 


\section{In vitro susceptibility of C. albicans}

Broth microdilution testing was performed in accordance with the guidelines in the Clinical \& Laboratory Standards Institute (CLSI) document M27-A3 [18]. Susceptibility was determined by the microbroth dilution method. Briefly, C. albicans strains were suspended in phosphate-buffered saline (PBS). CHT was dissolved in DMSO (Sigma, MO, USA) and diluted in RPMI 1640 medium supplemented with L-glutamine and buffered to pH 7.0 with $0.165 \mathrm{M}$ morpholine propanesulfonic acid (Sigma). The compounds were tested at concentrations of $0.125-64 \mathrm{mg} / \mathrm{l}$. As growth and sterility controls, RPMI alone was used, without the addition of the compound and solvent. In addition, a control for the toxicity of the solvent, a culture was inoculated with DMSO. Fluconazole (Sigma) and amphotericin B (Sigma) were included as positive antifungal controls and were tested at concentrations of $0.125-64 \mathrm{mg} / \mathrm{l}$. The plates were incubated at $35^{\circ} \mathrm{C}$ for $48 \mathrm{~h}$. Three independent experiments were performed in duplicate.

\section{Antibiofilm activity}

C. albicans biofilm

An inoculum of $C$. albicans SC5314 was adjusted to $10^{6}$ cells $/ \mathrm{ml}$ in RPMI medium and $2 \mathrm{ml}$ of the inoculum was distributed in 24-well microplates containing sterile silicone pads. The microplates were incubated at $37^{\circ} \mathrm{C}$ under agitation of $80 \times \mathrm{g}$. At 24 and $48 \mathrm{~h}$, the medium was removed and the wells were washed two-times with PBS, then $2 \mathrm{ml}$ of RPMI 1640 medium was added to the wells. At $72 \mathrm{~h}$ the plates were washed again and $2 \mathrm{ml}$ of RPMI containing CHT in concentrations equal to the MIC were added to the biofilms. Plates were incubated for $24 \mathrm{~h}$ then washed with PBS and the silicone pads were placed at room temperature to dry before measuring the biofilm dry weight [19]. Six replicates were made in two independent experiments.

\section{Reduction assay with XTT in biofilm of Cryptococcus}

Evaluation of Cryptococcus biofilm was performed according to Martinez \& Casadevall [20] with modifications. C. gattii L27/01 and C. neoformans ATCC24067 were grown in Sabouraud dextrose broth for $24 \mathrm{~h}$ at $37^{\circ} \mathrm{C}$ with agitation at $120 \times \mathrm{g}$. Cells were then collected by centrifugation, washed twice with PBS, and suspended at $10^{7} \mathrm{cell} / \mathrm{s} / \mathrm{ml}$ in minimal medium. One hundred microliters of the suspension were added into wells of 96-well microplates and incubated at $37^{\circ} \mathrm{C}$. After $24 \mathrm{~h}$, the wells containing biofilms were washed three-times with $0.05 \%$ Tween 20 (Sigma-Aldrich, MO, USA) in Tris-buffered saline to remove nonadhered cryptococcal cells. Then, we added $100 \mu \mathrm{l}$ of minimal medium and incubated the plates for another $24 \mathrm{~h}$. Subsequently, the supernatant was removed and the plates were washed, and after that plates were replaced with medium containing CHT compound according to MIC values (1 mg/l) to both Cryptococcus strains previously determined by Sá et al. [16] for 24 h.

After this period the plates were again washed in order to remove cells not adhered to the biofilm, and metabolic activity of the attached cells was measured with 2,3-bis(2-methoxy-4-nitro-5-sulfophenyl)-5((phenylamino)carbonyl)-2H-tetrazolium hydroxide (XTT, Sigma) according with Meshulam et al. [21]. All assays were carried out in ten replicates.

\section{Inhibition of C. albicans adhesion in human buccal epithelial cell}

A $1 \mathrm{ml}$ suspension of $10^{6}$ cells $/ \mathrm{ml}$ of the 12 C. albicans isolates was added to $4 \mathrm{ml}$ of Saboraud Dextrose Broth (SDB) containing CHT or fluconazole at concentrations equal to the MIC, and a positive control was prepared containing medium without drug. The samples were incubated at $37^{\circ} \mathrm{C}$ for $1 \mathrm{~h}$ with agitation at $120 \times$ g. After the treatment period, cells were washed twice with PBS before suspension in $3 \mathrm{ml}$ of PBS.

The method described by Kimura and Pearsall [22] with modifications was used to test cell adhesion. Human buccal epithelial cells (HBEC) from healthy adult volunteers were collected in the morning. The HBEC suspension was washed four-times in PBS with centrifugation at $3000 \times g$ for $10 \mathrm{~min}$ and resuspended in PBS, at a final concentration of $10^{5}$ cells $/ \mathrm{ml}$. Then, $0.5 \mathrm{ml}$ of HBEC suspension and $0.5 \mathrm{ml}$ of yeast suspension were mixed gently and incubated on a rotary shaker at $37^{\circ} \mathrm{C}$ for $1 \mathrm{~h}$. At the end of this period, cells were filtered on polycarbonate filter with pore size $12 \mu \mathrm{m}$ and washed with $100 \mathrm{ml}$ of PBS to remove non-adherent yeast cells. After, the filter was removed and put on a glass slide. Preparations were air dried, fixed with heat, and stained with crystal violet. Yeasts adherent to the buccal cells were counted under a light microscope $(40 \times)$. In each slide, we examined 50 HBEC and counted the number of associated fungal cells. 


\section{Survival curve of Galleria mellonella}

For the model of $C$. albicans infection in G. mellonella studies were performed as previously described by Fuchs et al. [23]. G. mellonella (Vanderhorst Wholesale, OH, USA) in the final larval stage was stored in the dark and used within 7 days from shipment. In addition, tests with Cryptococcus spp. had been performed using G. mellonella in the final larval stage and studies were performed as previously described by Mylonakis et al. [24]. G. mellonella were obtained from Laboratory of Microbiology and Immunology of Univ Estadual Paulista (Unesp, São José dos Campos, São Paulo, Brazil).

C. albicans SC5314 was cultured in YPD broth (1\% yeast extract, $2 \%$ bacto-peptone, $2 \%$ dextrose) at $30^{\circ} \mathrm{C}$ overnight and C. gattii L27/01 and C. neoformans ATCC24067 were prepared by culturing the cells in YPD broth at $37^{\circ} \mathrm{C}$ for $48 \mathrm{~h}$ and then all yeasts were washed three-times with PBS. Yeast cells were counted using a hemocytometer. A Hamilton syringe was used to inject yeasts in a volume of $10 \mu \mathrm{l}$ into the hemocele of each larva via the last left proleg. Each larva received $10 \mu \mathrm{l}$ containing $10^{6}$ cells/larva of C. albicans and C. neoformans and $10^{7}$ cells/larvae of C. gattii into the hemocele.

Experimental groups received $10 \mu \mathrm{l}$ of CHT solubilized in a vehicle $(10 \mathrm{mg} / \mathrm{kg})$ and injected at the last right proleg. Two control groups were included in all experiments: one group was inoculated with PBS to account for any killing due to physical trauma, and the other received no injection as a control for general viability. After injections, larvae were incubated in Petri plates at $37^{\circ} \mathrm{C}$ and monitored for survival daily.

\section{Murine models \\ Animals}

C57BL/6 mice, approximately 6-8 weeks in age and 20-25 g were supplied by the Biological Center of the Federal University of Minas Gerais (Cebio, UFMG, Belo Horizonte, Minas Gerais, Brazil). Male mice were used in the cryptococcosis model and female in the systemic candidiasis model. Tests in the animal models were in accordance with the Ethics Committee for Animal Experimentation (CEUA/UFMG), protocol n ${ }^{\circ}$ 221/2013.

\section{Cryptococcosis}

The animals were infected intratracheally with $10^{5}$ yeasts of C. gattii L27/01 in sterile saline $(\mathrm{NaCl} 0.85 \%)$. After $24 \mathrm{~h}$ of infection, the mice were divided into five experimental groups ( $\mathrm{n}=6$ animals/group) as following: control not infected; control with infected animals without treatment; infected animals treated with fluconazole at $10 \mathrm{mg} / \mathrm{kg} /$ day; infected animals treated with CHT at $10 \mathrm{mg} / \mathrm{kg} /$ day; infected animals treated with CHT at $50 \mathrm{mg} / \mathrm{kg} /$ day. All treated groups received daily, intraperitoneally, CHT (10 or $50 \mathrm{mg} / \mathrm{kg} /$ day) or fluconazole $(10 \mathrm{mg} / \mathrm{kg} /$ day $)$ injection. The treatment was performed for 15 days and survival was monitored until the animals died. The CHT compound was diluted in polyethylene glycol $400(20 \%)$, Tween $80(0.05 \%)$ and PBS, and fluconazole was prepared in PBS.

After survival analysis another experiment was performed for colony-forming unit (CFU) recovery. Then, animals were infected and treated for 15 days with CHT or fluconazole $(10 \mathrm{mg} / \mathrm{kg} /$ day $)$. At the designated time end point, animals were anesthetized and euthanized. Brain and lung tissues were harvested to evaluate the fungal burden. The collected tissue was homogenized in PBS, serially diluted, and plated on YPD to determine the number of CFUs [25]. Three independent experiments were carried out with six animals per group.

\section{Systemic candidiasis}

Mouse model of systemic candidiasis was established according to a previously described method by Wong et al. [26], with modifications. The mice were injected via the tail vein with $30 \mu \mathrm{l}$ cell suspension of C. albicans SC5314, resulting in $10^{5}$ yeasts per animal $3 \mathrm{~h}$ before the start of antifungal treatment. Treatments were given to the mice via intraperitoneal injection at 3, 8, 24, 36, 48, 60, 72 and $84 \mathrm{~h}$ after infection with fluconazole $(10 \mathrm{mg} / \mathrm{kg})$ or CHT (10 mg/kg; $\mathrm{n}=6$ animals/group). The CHT compound and fluconazole were diluted according to previously described for cryptococcosis in vivo assay. Two control groups with infected and not-infected animals were also used. All of the mice were sacrificed after $96 \mathrm{~h}$, and the kidneys were removed, weighed and then homogenized in PBS, and the homogenates were serially diluted before plating on Sabouraud dextrose medium plates. The plates were incubated at $37^{\circ} \mathrm{C}$ for $48 \mathrm{~h}$, and fungal burden was expressed as the ratio of CFU/g of kidney. 


\begin{tabular}{|c|c|c|c|}
\hline \multirow[t]{2}{*}{ Fungal species } & \multirow[t]{2}{*}{ Strains } & \multicolumn{2}{|c|}{ MIC (mg/l) of Candida strains } \\
\hline & & CHT & FLZ \\
\hline Candida albicans & SC5314 & 4 & 1 \\
\hline C. albicans & ATCC 18804 & 2 & 2 \\
\hline C. albicans & UFMG-CM-Y6071 & 4 & $>64$ \\
\hline C. albicans & UFMG-CM-Y6073 & 64 & 4 \\
\hline C. albicans & UFMG-CM-Y6074 & 2 & 2 \\
\hline C. albicans & UFMG-CM-Y6075 & 1 & 8 \\
\hline C. albicans & UFMG-CM-Y6076 & 4 & $>64$ \\
\hline C. albicans & UFMG-CM-Y6077 & 4 & $>64$ \\
\hline
\end{tabular}

CHT: 2-(2-(cyclohexylmethylene)hydrazinyl)-4-phenylthiazole; FLZ: Fluconazole; MIC: Minimal inhibitory concentration

\section{Statistical analysis}

Percent survival and killing curves of $G$. mellonella were plotted and statistical analysis was performed by the Kaplan-Meier test using GraphPad Prism statistical software (GraphPad Software, Inc., CA, USA). Statistical analysis of results of antibiofilm activity, inhibition of $C$. albicans adhesion and murine tests used Newman-Keuls Multiple and the Student's $t$-test using GraphPad Prism and p-value $\leq 0.05$ was considered significant and results were expressed as mean \pm standard error of the mean.

\section{Results}

CHT has demonstrated inhibition against 24 isolates of Cryptococcus spp. [16]. We expanded this, finding that CHT is also efficacious against $C$. albicans. Susceptibility tests with $C$. albicans isolates resulted in MIC values ranging from 1 to $4 \mathrm{mg} / \mathrm{l}$ for most isolates, but two clinical isolates, UFMG-CM-Y6080 and UFMG-CM-Y6073, did not showed susceptibility at concentrations up to $64 \mathrm{mg} / 1$ of CHT. Among six C. albicans clinical isolates examined, we observed fluconazole resistance with MIC values $>64 \mathrm{mg} / 1$ (Table 1). Only one clinical strain of $C$. albicans exhibited dual resistance to both CHT and fluconazole. Sá et al. [27] showed evidence that CHT target action is related to interference in antioxidant fungal system and here in this work we show that in addition to this $\mathrm{CHT}$ is also capable of inhibiting $C$. albicans adhesion in HBEC and shows an indication in reducing of biofilms.

Although we knew that CHT could inhibit planktonic cells as assessed by our previous observation of the low MIC of $1 \mathrm{mg} / \mathrm{l}$ found against Cryptococcus spp. [16], we interrogated the ability of CHT to inhibit biofilm formation. In order to verify the ability of CHT to inhibit the biofilm states sessile cells in biofilms formed by C. gattii L27/01 and C. neoformans ATCC24067 were challenged. Results of these experiments showed a significant reduction of the metabolic activity of yeasts in biofilms formed by $C$. gattii $(44.2 \%)$ and $C$. neoformans (30.8\%) after treatment with CHT compared with control biofilm without treatment ( $<<0.0001$ to both) (Figure 2).

Since CHT was effective in reducing Cryptococcal biofilms, we further investigated the capacity to inhibit Candida biofilm. We found a reduction in dry weight of C. albicans biofilm (73.8\%) after treatment with CHT (Figure 3A), with a significant difference between control and treatment $(p=0.0195)$. Successful biofilm formation is dependent upon the capacity for cell adhesion in order to anchor fungi to a device or tissue. One of the most impacted sites by biofilm formation is the oral cavity [28,29]. The inclusion of CHT resulted in a $75.12 \pm 12 \%$ reduction in $C$. albicans to HBEC (Figure 3B). The reduction of adhesion capacity was similar for CHT and fluconazole $(60.5 \pm 19 \%)$ treatment. The culmination of biofilm disturbance suggests that CHT can inhibit biofilm formation, a normally effective fungal strategy of invading a host and evading therapeutic damage.

Based on these in vitro findings, we studied the efficacy of CHT in vivo. G. mellonella was selected as an invertebrate infection model for its susceptibility to various fungal pathogens [30]. The toxicity of the compound was tested in G. mellonella larvae and we found the $\mathrm{LD}_{50}>10 \mathrm{mg} / \mathrm{kg}$ in the absence of infection, thus, no significant toxicity of this thiazole derivative in the larvae (Figure 4A). After treatments with CHT, G. mellonella larvae infected 

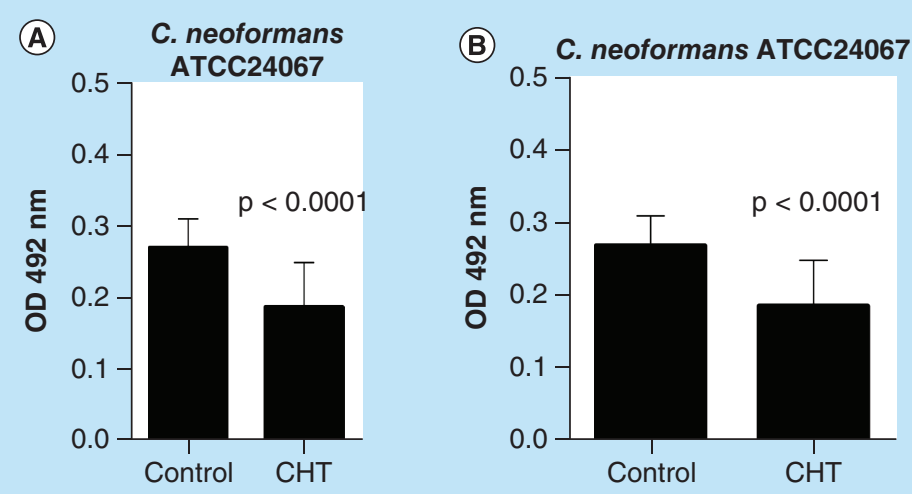

Figure 2. Antifungal activity of 2-(2-(cyclohexylmethylene)hydrazinyl)-4-phenylthiazole in biofilms formed by Cryptococcus spp. by reduction assay with 2,3-bis(2-methoxy-4-nitro-5-sulfophenyl)-5-((phenylamino)carbonyl)-2H-tetrazolium hydroxide. (A) Cryptococcus gattii L27/01. (B) Cryptococcus neoformans ATCC24067. Statistical analyses were performed by Student's $t$-test. Results were expressed as mean \pm standard error of the mean.

(A)

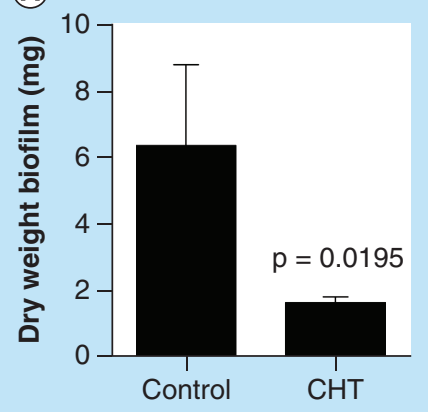

(B)

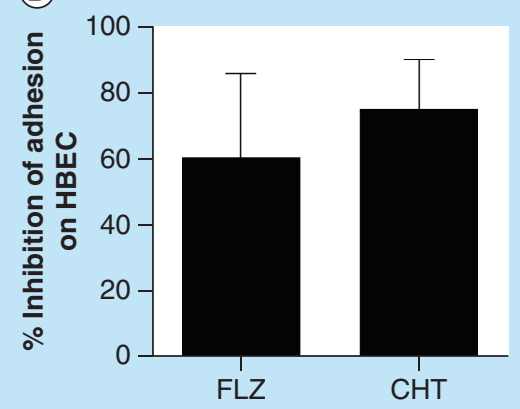

Figure 3. Evaluation of the inhibition of Candida albicans virulence factors after treatment with 2-(2-(cyclohexylmethylene)hydrazinyl)-4-phenylthiazole at MIC. (A) Evaluation of the reduction in biomass of biofilm formed by C. albicans SC5314 after treatment with CHT. Statistical analyses were performed by Newman-Keuls multiple comparison test. (B) Percent inhibition of $\mathrm{C}$. albicans adhesion treated for $1 \mathrm{~h}$ with $\mathrm{CHT}$ and fluconazole at MIC on human buccal epithelial cells. Data representative of two independent experiments with 12 isolates of $C$. albicans. The control group without treatment represents $100 \%$ of adhesion of yeasts on human buccal epithelial cells $(0 \%$ of inhibition). There were no significant differences between fluconazole or CHT treatment $(p>0.05)$. Statistical analysis was performed by Newman-Keuls multiple comparison test. Results were expressed as mean \pm standard error of the mean.

CHT: 2-(2-(cyclohexylmethylene)hydrazinyl)-4-phenylthiazole; FLZ: Fluconazole; HBEC: Human buccal epithelial cells.

with C. gattii L27/01 or C. neoformans ATCC24067 exhibited significantly prolonged survival (Figure 4C).

A G. mellonella infection model was also used to test the therapeutic potential against Candida. The CHT compound showed significantly prolonged larval survival (Figure 4D). The two doses tested were efficient, but the best dose was $5 \mathrm{mg} / \mathrm{kg}$ resulting in $>70 \%$ of survival after 6 days of infection, while, in the same period, a dose equal to $10 \mathrm{mg} / \mathrm{kg}$ provided only $40 \%$ larval survival. Thus the broad-spectrum antifungal character of $\mathrm{CHT}$ is legitimized in an in vivo infection model.

The encouraging results from the insect model prompted us to extend our evaluation into a mammalian infection model. In a murine model of cryptococcosis caused by $C$. gattii the groups of animals infected and treated with fluconazole and $\mathrm{CHT}$ at 10 and $50 \mathrm{mg} / \mathrm{kg} /$ day exhibited a longer survival in comparison to control animals without treatment $(\mathrm{p}<0.05)$ (Figure 5A). It was observed that there were still live animals up to day 37 of CHT treatment and in the fluconazole group all animals had died by day 28 , whereas in the group without treatment, all 


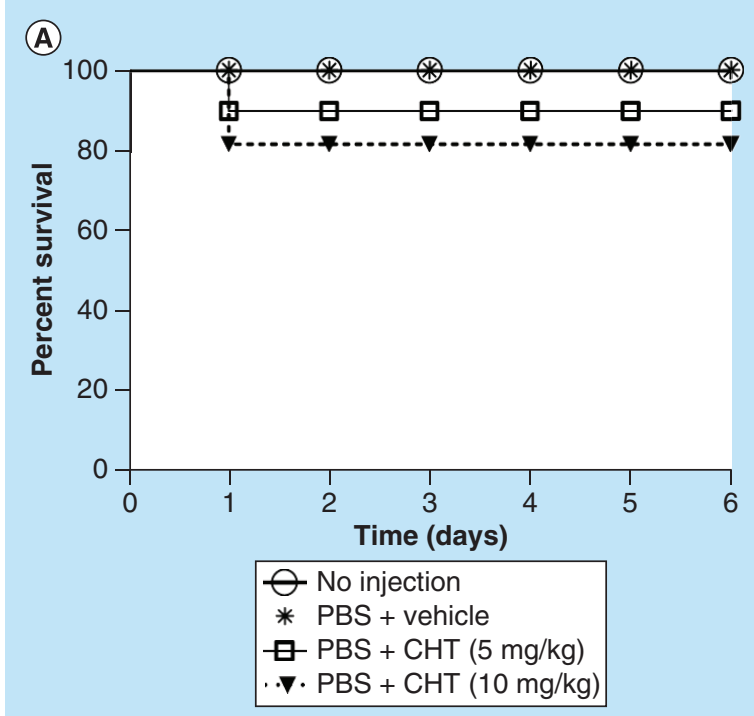

(B)

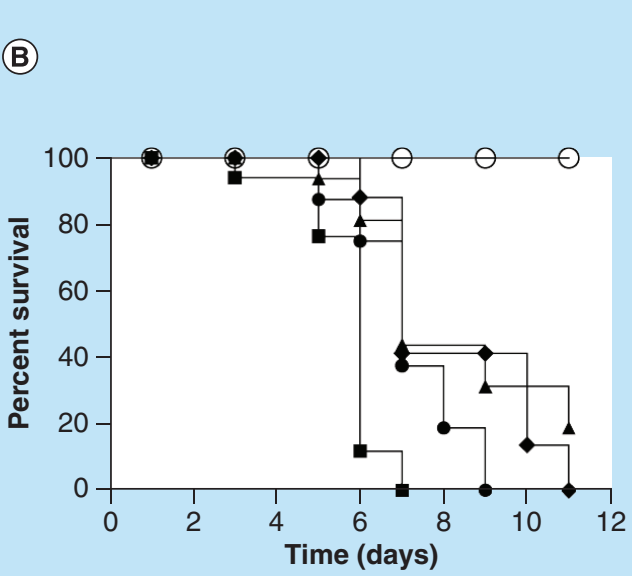

(c)

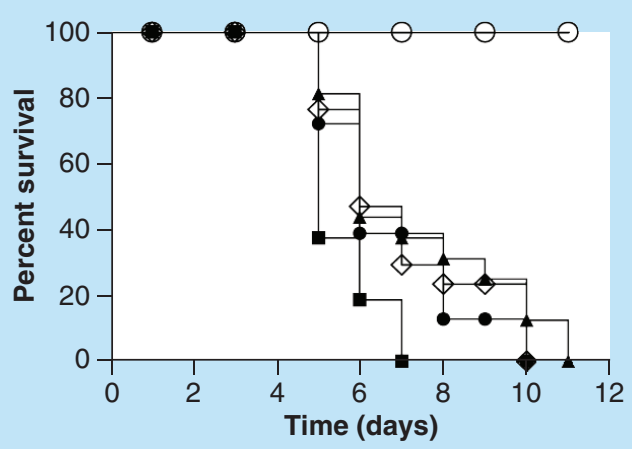

(D)

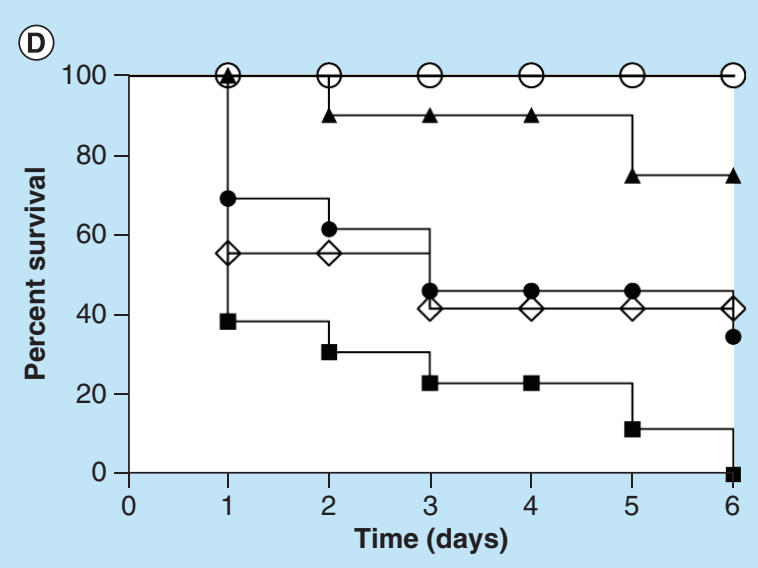

$$
\begin{aligned}
& \ominus \text { No injection } \\
& - \text { C. neoformans + vehicle } \\
& - \text { C. neoformans + CHT }(5 \mathrm{mg} / \mathrm{kg}) \\
& \leftarrow \text { C. neoformans + CHT }(10 \mathrm{mg} / \mathrm{kg}) \\
& \ominus \text { C. neoformans + FLZ }(10 \mathrm{mg} / \mathrm{kg})
\end{aligned}
$$

$\ominus$ No injection

- C. gattii + vehicle

- C. gattii + CHT $(5 \mathrm{mg} / \mathrm{kg})$

- C. gattii + CHT $(10 \mathrm{mg} / \mathrm{kg})$

- C. gattii + FLZ $(10 \mathrm{mg} / \mathrm{kg})$ 

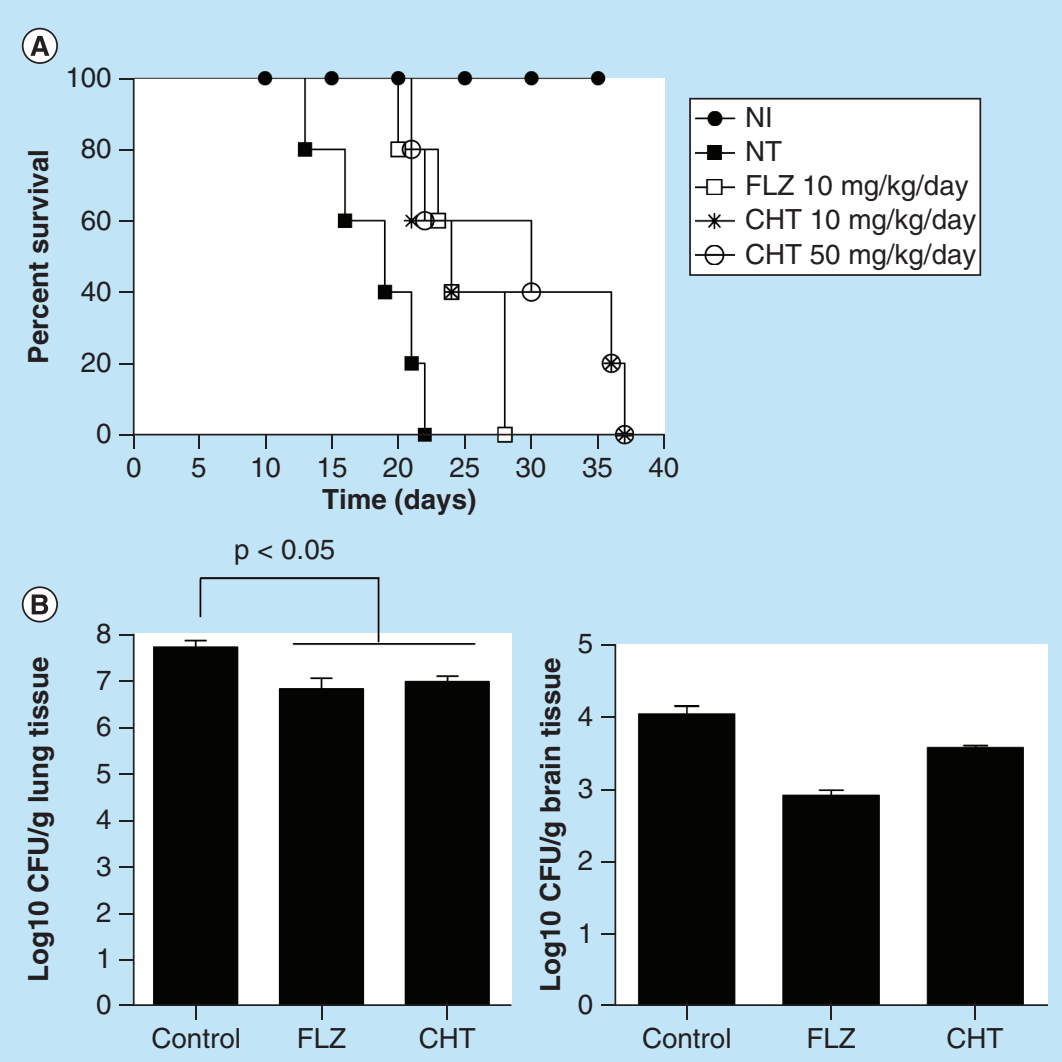

Figure 5. Evaluation of 2-(2-(cyclohexylmethylene) hydrazinyl)-4-phenylthiazole treatment in murine model. (A) Survival curve of the male C57BL/6 mice infected with Cryptococcus gattii L27/01 (10 $0^{5}$ yeast) and treated with CHT and FLZ. The three treatments significantly increased survival at $\mathrm{p}<0.05$ to FLZ $10 \mathrm{mg} / \mathrm{kg} / \mathrm{day}, \mathrm{CHT}$ at 10 and $50 \mathrm{mg} / \mathrm{kg} /$ day (Kaplan-Meier test). Representative data from two independent experiments with $n=5$. (B) Recovery of colony-forming units from lung and brain from male C57BL/ 6 mice infected with C. gattii L27/01 (105 yeast) and treated CHT and FLZ for 15 days. There was a significant reduction in colony-forming units for the lung $(p<0.05)$, but not in the brain $(p>0.05)$ for both treatments. Statistical analyses were performed by Newman-Keuls multiple comparison test. Results were expressed as mean \pm standard error of the mean.

CFU: Colony-forming unit; CHT: 2-(2-(cyclohexylmethylene)hydrazinyl)-4-phenylthiazole; FLZ: Fluconazole; NI: Control not infected; NT: Control with infected animals without treatment.

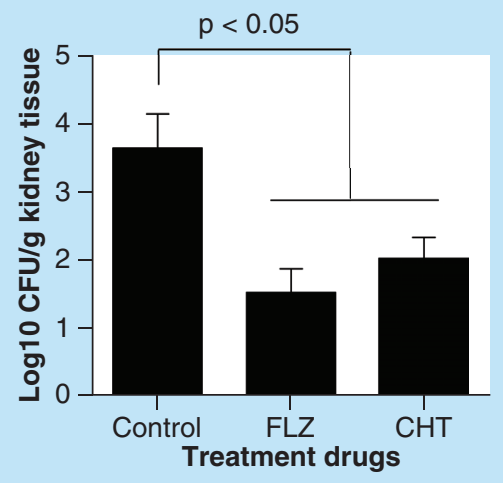

Figure 6. Evaluation of 2-(2-(cyclohexylmethylene)hydrazinyl)-4-phenylthiazole treatment in murine model of systemic candidiasis. Recovery of colony-forming units from kidneys from female C57BL/ 6 mice infected with Candida albicans SC5314 ( $10^{5}$ yeasts) and treated

2-(2-(cyclohexylmethylene)hydrazinyl)-4-phenylthiazole and fluconazole. Statistical analyses were performed by Newman-Keuls multiple comparison test. Results were expressed as mean \pm standard error of the mean.

CFU: Colony-forming unit; $\mathrm{CHT}$ :

2-(2-(cyclohexylmethylene)hydrazinyl)-4-phenylthiazole; FLZ: Fluconazole.

$>0.05)$. In murine model of systemic candidiasis the fungal burden of the kidneys were lower in mice that received treatment with CHT or fluconazole than in mice that not received treatment (Figure 6). 


\section{Discussion}

The heterocyclic thiazole CHT was previously evaluated in in vitro assays by Sá et al. [16] and showed interesting results against $C$. gattii and $C$. neoformans. In the present work we expand the fungal pathogens susceptible to the compound to include C. albicans. Our interrogation was able to correlate in vitro activity with the efficacy of CHT treatment in animal models.

In the biofilm assays with Cryptococcal species and C. albicans we observed that CHT compound were also active against sessile cells aggregated as biofilms formed by these species, which is a key factor in fungal pathogenesis [31]. The biofilm formation phases are very similar between Cryptococcus spp. and C. albicans [32]. Biofilms represent the most common mode of growth of microorganisms in nature, a state that presumably allows yeasts cells to both survive hostile environments, making the cells more resistant to host immune mechanisms and drug therapy [33].

C. albicans biofilm is formed through coordinated phases such as early, intermediate and maturation stages [32]. Especially in early growth phase yeasts need to be strongly adhered to the surfaces. Then, we observed that CHT treatment also interferes with the adhesion capacity of Candida yeasts to HBEC, which is closely related to biofilm construction. Adhesion of C. albicans to host cells is essential to colonization, biofilm formation and survival in the host, and this process requires the interaction of Candida adhesins with host cell receptors [34].

In view of this promising in vitro activity of the CHT compound we tested it in in vivo models. Initially we evaluated CHT in the invertebrate model with G. mellonella infected with C. gattii, C. neoformans or C. albicans. Treatment of larvae with CHT resulted in increased larval survival. Based on our results we believe that the CHT compound initiates some accumulation of harmful events on fungal cell that causes a failure in protecting the fungi from executing the virulence factors, such as $C$. albicans adhesion (which may be related to the $C$. albicans biofilm development).

In a previous work [16] we had demonstrated that there was no toxicity to mammalian cells, and recently we verified that $\mathrm{CHT}$ is nontoxic to human erythrocytes [27]. These findings indicate that CHT has potential in a murine model, which is considered the gold standard for studying pathogenesis, quantifying virulence, and analyzing the efficacy of antifungal drugs [30].

Thus, in the present work we observed that the CHT compound was efficient in prolonging the survival of mice infected with C. gattii L27/01 and C. albicans SC5314 similarly to fluconazole. The isolate $\mathrm{L} 27 / 01$ of $C$. gattii presents an intermediate virulence phenotype in mice and has been used in several types of studies in murine cryptococcosis model $[25,30,35]$. The reference isolate SC5314 of $C$. albicans presents high virulence and has been used in several works [36,37]. Jaen-Chen et al. [36] showed that intravenous injection of SC5314 results in mortality of animals between 8 and 35 days postinfection according to inoculum dosage.

Santos et al. [38] showed that C57BL/6 mice infected with L27/01 treated with fluconazole at $10 \mathrm{mg} / \mathrm{kg} / \mathrm{day}$ had a significant increase in survival similar to the dose of $75 \mathrm{mg} / \mathrm{kg} /$ day. In addition to promoting a significant reduction in lung fungal load, which can be correlated with our work, confirming that the dose of $10 \mathrm{mg} / \mathrm{kg} / \mathrm{day}$ is sufficient to generate significant effects and therefore can be used for comparison with experimental compounds.

The intravenous infection for systemic candidiasis is well characterized and reproducible model, and allows yeasts to spread rapidly throughout the body of the host; however, usually the infection is controlled in many organs but progresses in the kidneys in the absence of efficient antifungal treatment [39]. In the present study we observed similar control of fungal burden from kidneys after treatments with CHT and fluconazole, which is used for the treatment of systemic candidiasis.

\section{Conclusion}

In the present work the efficiency of a new phenylthiazole was associated with important fungicidal activity, ability to inhibit virulence factors via inhibition of $C$. albicans adhesion in human buccal epithelial cell, and showed evidence of reduced C. albicans and Cryptococcus biofilms. We believe that all these processes observed in vitro are closely related to the significant results observed in the murine model of cryptococcosis and candidiasis. This study presents evidence of CHT's therapeutic potential.

\section{Future perspective}

The substance presented in this study has potential for systemic use in the treatment of cryptococcosis and candidiasis, and also in topical use for oral candidiasis. The preliminary results reinforce the possibility that this compound, after all the necessary tests and clinical trials, could be used as a prototype for the development of a new antifungal. 
Executive summary

- 2-(2-(cyclohexylmethylene)hydrazinyl)-4-phenylthiazole (CHT) is a thiazole derivative.

- $\mathrm{CHT}$ is active against Candida and Cryptococcus.

- In a previous study the mechanism of action of $\mathrm{CHT}$ was related open in the fungal antioxidant system.

- $\mathrm{CHT}$ works well in both suspension and biofilm cells.

- In Candida, CHT inhibits adhesion ability.

- In Galleria mellonella, treatment with CHT was effective for candidiasis and cryptococcosis model.

- In systemic use, CHT is efficient for the treatment of cryptococcosis and candidiasis in a murine model.

- CHT has shown activity against Candida albicans against cells in suspension and also in biofilm, besides affecting the ability of these yeasts to adhere to oral cells.

- CHT was also active in biofilms of Cryptococcus gattii and Cryptococcus neoformans.

- The experimental compound increased the survival of G. mellonella larvae infected with C. albicans, C. gattii and C. neoformans.

- In the murine model, CHT was able to reduce the fungal load of the lungs of mice infected with C. gattii.

- $\mathrm{CHT}$ was also efficient in reducing the fungal load in the kidneys of animals with systemic candidiasis.

Authors' contributions

N P Sá did study design, development and methodology of all antifungal assay, data analysis/interpretation and writing all the manuscript. C M Lima looked after development and methodology of antifungal assay. J R A dos Santos and M C Costa looked after development and methodology of murine model and data analysis/interpretation. P P de Barros looked after development and methodology of biofilm assay; J C Junqueira looked after study design of biofilm assay and data analysis/interpretation. J A Vaz looked after development and methodology of compound synthesis. R B Oliveira looked after study design, development and methodology of compound synthesis and manuscript revision. B B Fuchs looked after study design and data analysis/interpretation of Galleria mellonella studies and manuscript revision. E Mylonakis looked after study design and data analysis/interpretation of G. mellonella studies and manuscript revision. C A Rosa looked after study design of antifungal assay and manuscript revision. D A Santos looked after study design, data analysis/interpretation of murine model and manuscript revision. S Johann looked after study design, data analysis/interpretation of all study and manuscript revision.

Financial \& competing interests disclosure

Funding was provided by a grant to B B Fuchs and E Mylonakis from the Brown/Brazil Initiative and by Conselho Nacional de Desenvolvimento Científico e Tecnológico (CNPq), Fundação de Amparo Pesquisa Estado de Minas Gerais (FAPEMIG) and Coordenação de Aperfeiçoamento de Pessoal de Nível Superior (CAPES). The authors have no other relevant affiliations or financial involvement with any organization or entity with a financial interest in or financial conflict with the subject matter or materials discussed in the manuscript apart from those disclosed.

No writing assistance was utilized in the production of this manuscript.

Ethical conduct of research

Tests in the animal models were in accordance with the Ethics Committee for Animal Experimentation (CEUA/UFMG), protocol $\mathrm{n}^{\circ}$ $221 / 2013$.

The authors state that they have obtained appropriate institutional review board approval or have followed the principles outlined in the Declaration of Helsinki for all human or animal experimental investigations. In addition, for investigations involving human subjects, informed consent has been obtained from the participants involved.

Open access

This work is licensed under the Creative Commons Attribution 4.0 License. To view a copy of this license, visit http://creativecommons.org/licenses/by/4.0/ 


\section{References}

Papers of special note have been highlighted as: • of interest; $\bullet \bullet$ of considerable interest

1. Casadevall A. Fungal virulence vertebrate endothermy, and dinosaur extinction: is there a connection? Fungal Genet. Biol. 42(2), 98-106 (2005).

2. Brown GD, Denning DW, Gow NA et al. Hidden killers: human fungal infections. Sci. Transl. Med. 4(165), 165rv13 (2012).

3. Schmiedel Y, Zimmerli S. Common invasive fungal diseases: an overview of invasive candidiasis, aspergillosis, cryptococcosis, and Pneumocystis pneumonia. Swiss Med. Wkly 146, w14281 (2016).

4. Park BJ, Wannemuehler KA, Marston BJ et al. Estimation of the current global burden of cryptococcal meningitis among persons living with HIV/AIDS. AIDS 23, 525-530 (2009).

5. Casadevall A, Cleare W, Feldmesser $\mathrm{M}$ et al. Characterization of a murine monoclonal antibody to Cryptococcus neoformans polysaccharide that is a candidate for human therapeutic studies. Antimicrob. Agents Chemother. 42, 1437-1446 (1998).

6. Menezes RP, Penatti MPA, Pedroso RS. Different culture media containing methyldopa for melanin production by Cryptococcus species. Rev. da Soc. Bras. de Med. Trop. 44(5), 591-594 (2011).

7. Rodrigues ML, Nimrichter L, Cordero RJB et al. Fungal polysaccharides: biological activity beyond the usual structural properties. Front Microbiol. 2, 171-174 (2011).

8. Pukkila-Worley R, Ausubel FM, Mylonakis E. Candida albicans infection of Caenorhabditis elegans induces antifungal immune defenses. PLoS Pathog. 7(6), e1002074 (2011).

9. Khaled H, Abu-Elteen Hamad MA. Changing epidemiology of classical and emerging human fungal infections: a review. Jord. J. Biol. Sci. 5(4), 215-230 (2012).

10. Allen UD. Antifungal agents for the treatment of systemic fungal infections in children. Paediatr. Child Health 15(9), 603-608 (2010).

11. Perfect JR, Dismukes WE, Dromer F et al. Clinical practice guidelines for the management of cryptococcal disease: 2010 update by the Infectious Diseases Society of America. Clin. Infect. Dis. 50, 291-322 (2010).

12. Srichatrapimuk S, Sungkanuparph S. Integrated therapy for HIV and cryptococcosis. AIDS Res. Ther. 13, $42-57$ (2010).

13. Fica AC. Tratamiento de infecciones fúngicas sistêmicas primeira parte: fluconazol, itraconazol y voriconazol. Rev. Chil. Infectol. 21, 26-38 (2004).

14. Kon AS, Grumach AS, Colombo A et al. Consenso em Criptococose. Rev. Soc. Bras. Med. Trop. 41(5), 524-544 (2008).

15. Revankar SG, Fu J, Rinaldi MG et al. Cloning and characterization of the lanosterol $14 \alpha$-demethylase (ERG11) gene in Cryptococcus neoformans. Biochem. Biophys. Res. Comm. 324, 719-728 (2004).

16. Sá NP, Lino CI, Fonseca NC et al. Thiazole compounds with activity against Cryptococcus gattii and Cryptococcus neoformans in vitro. Eur. J. Med. Chem. 102, 233-242 (2015).

-. The first work that presents the synthesis and antifungal activity of compound 2-(2-(cyclohexylmethylene)hydrazinyl)-4-phenylthiazole (previously named 1d). Shows evidence of the activity of this compound against Cryptococcus gattii and Cryptococcus neoformans.

17. Hagen F, Khayhan K, Theelen B et al. Recognition of seven species in the Cryptococcus gatti/Cryptococcus neoformans species complex. Fungal Genet. Biol. 78, 16-48 (2015).

18. CLSI. Reference method for broth dilution antifungal susceptibility testing of yeasts; approved standard (3rd Edition). Clinical and Laboratory Standards Institute, PA, USA. CLSI document M27-A3 (2008).

19. Breger J, Fuchs BB, Aperis G et al. Antifungal chemical compounds identified using a C. elegans pathogenicity assay. PLoS Pathog. 3(2), e18 (2007).

20. Martinez LR, Casadevall A. Specific antibody can prevent fungal biofilm formation and this effect correlates with protective efficacy. Inf. Immun. 73(10), 6350-6362 (2005).

-• Of considerable interest in the execution of this work, because it is one of the few papers that studies the formation of biofilms by Cryptococcus. Presents the ability of Cryptococcus to form a biofilm and describe methodologies of good reproducibility.

21. Meshulam T, Levitz SM, Christin L et al. A simplified new assay for assessment of fungal cell damage with the tetrazolium dye, (2,3)-bis-(2-methoxy-4-nitro-5-sulphenyl)-(2H)-tetrazolium-5-carboxanilide (XTT). J. Infect. Dis. 172, 1153-1156 (1995).

22. Kimura LH, Pearsall NN. Adherence of Candida albicans to human buccal epithelial cells. Infect. Immun. 21(1), 64-68 (1978).

23. Fuchs BB, O’Brien E, El Khoury JB et al. Methods for using Galleria mellonella as a model host to study fungal pathogenesis. Virulence 1(6), 475-482 (2010).

- An important work that provides relevant arguments and information about the use of invertebrate Galleria mellonella in the study of fungal diseases.

24. Mylonakis E, Moreno R, El Khoury JB et al. Galleria mellonella as a model system to study Cryptococcus neoformans pathogenesis. Infect. Immun. 73, 3842-3850 (2005). 
25. Santos JRA, Holanda RA, Frases $S$ et al. Fluconazole alters the polysaccharide capsule of Cryptococcus gattii and leads to distinct behaviors in murine cryptococcosis. PLoS ONE 9(11), e112669 (2014).

26. Wong SSW, Kao RYT, Yuen KY et al. In vitro and in vivo activity of a novel antifungal small molecule against Candida infections. PLoS ONE 9(1), e85836 (2014).

-• Clearly presents the study of murine candidiasis in a systemic and oral model, adapted for the purpose of the present study to investigate the efficacy of CHT treatment in these two models.

27. Sá NP, Lima CM, Lino CI et al. Heterocycle thiazole compounds exhibit antifungal activity through increase in the production of reactive oxygen species in the Cryptococcus neoformans - Cryptococcus gattii species complex. Antimicrob. Agents Chemother. 61(8), e02700-e02716 (2017).

- Particularly interesting work because it presents relevant information about the action mechanism of 2-(2-(cyclohexylmethylene)hydrazinyl)-4-phenylthiazole compound.

28. Mathe L, Van Dijck P. Recent insights into Candida albicans biofilm resistance mechanisms. Curr. Genet. 59, 251-264 (2013).

29. Montelongo-Jauregui D, Srinivasan A, Ramasubramanian AK et al. An in vitro model for oral mixed biofilms of Candida albicans and Streptococcus gordonii in synthetic saliva. Front. Microbiol. 7, 686-696 (2016).

30. Binder U, Maurer E, Lass-Florl C. Galleria mellonella: an invertebrate model to study pathogenicity in correctly defined fungal species. Fungal Biol. 120, 288-295 (2016).

31. Gauwerky K, Borelli C, Korting HC. Targeting virulence: a new paradigm for antifungals. Drug Discov. Today 14(3-4), 214-222 (2009).

32. Chandra J, Kuhn DM, Mukherjee PK et al. Biofilm formation by the fungal pathogen Candida albicans: development, architecture, and drug resistance. J. Bacteriol. 183(18), 5385-5394 (2001).

33. Bergin D, Reeves EP, Renwick J et al. Superoxide production in Galleria mellonella hemocytes: identification of proteins homologous to the NADPH oxidase complex of human neutrophils. Infect. Immun. 73(7), 4161-4170 (2005).

34. Höfs S, Mogavero S, Hube B. Interaction of Candida albicans with host cells: virulence factors, host defense, escape strategies, and the microbiota. J. Microbiol. 54(3), 149-169 (2016).

- Reflects on the various virulence factors exhibited by the yeast Candida albicans.

35. Ferreira GF, Santos JRA, Costa MC et al. Heteroresistance to itraconazole alters the morphology and increases the virulence of Cryptococcus gattii. Antimicrob. Agents Chemother. 59, 4600-4609 (2015).

36. Jae-Chen S, Young-Joo J, Seon-Min P et al. Mechanism underlying renal failure caused by pathogenic Candida albicans infection. Biomed. Rep. 3(2), 179-182 (2015).

37. Staab JF, Datta K, Rhee P. Niche-specific requirement for hyphal wall protein 1 in virulence of Candida albicans. PLoS ONE 8(11), e80842 (2013).

38. Santos JR, Holanda RA, Frases $\mathrm{S}$ et al. Fluconazole alters the polysaccharide capsule of Cryptococcus gattii and leads to distinct behaviors in murine cryptococcosis. PLoS ONE 9(11), e112669 (2014).

39. Szabo EK, MacCallum DM. The contribution of mouse models to our understanding of systemic candidiasis. FEMS Microbiol. Lett. 320(1), 1-8 (2011). 